\title{
Control Strategy Research and Application Based on the Impact Analysis of PFC to AGC
}

\author{
Jun $\mathrm{Li}^{1, \mathrm{a}^{*}}$, Suhong Chen ${ }^{1}$, Hui Zhang ${ }^{2}$, Xun Zhang ${ }^{3}$ and Chaofeng $\mathrm{Wu}^{4}$ \\ ${ }^{1}$ State Grid Shandong Electric Power Research Institute, Jinan 250002, China \\ 2Dispatching \& Control Center, Shandong Electric Power Corporation, Jinan 250001, China \\ ${ }^{3}$ Huadian Zibo Thermal Power Co., Ltd., Zibo 255054, China \\ ${ }^{4}$ Huadian Tengzhou Xinyuan Thermoelectric Co., Ltd., Tengzhou 277500, China \\ alijun_sdu@hotmail.com
}

Keywords: automatic power generation control; primary frequency compensation; control strategy; performance standard; logic optimization

\begin{abstract}
With the rapid development of ultra-high voltage grid and interconnections of regional power network, it brings adverse influence on frequency control of the power system. The stability of power grid frequency mainly depends on primary frequency compensation (PFC) and automatic power generation control (AGC) of thermal power units. By analyzing the interaction between them and the actual action response to frequency of accidents, the control strategy were studied. By transformation and logic optimizations, the proposed control strategy can significantly reduce the number of PFC malfunction and effectively guarantee the quality of the AGC control.
\end{abstract}

\section{Introduction}

Along with the development of Ultra High Voltage Power Grid, grid operation in China has put forward higher stability requirements. As we known, power grid frequency is one of important indicators of power quality, it reflects the balance between the actual electricity usage and generation of active power, and it is an important control parameter of the power system operation. The frequency stability of the electric network is one of the most primary conditions of power system normal running. As a crucial basic indicator, mains frequency demands a balance between generating and power utilization. Currently, frequency compensation is mainly used for thermal power units. With the development of science and technology, some new electronic equipment and precision processing equipment put forward higher request to power grid frequency, the frequency fluctuations will lead to lower product quality or equipment damage. According to the IEEE 4461995 standard and BS EN50160:1995 standard, $\pm 0.5 \mathrm{~Hz}$ is the maximum tolerance of the frequency fluctuations of many devices.

The primary frequency compensation, refers to the grid frequency deviation from the rating, control system of unit in the grid automatically control the increase or decrease of the unit active power, to limit the changes of grid frequency, so that the grid frequency maintain the stability of automatic control process. The static frequency modulation characteristic of electric network is the foundation of frequency stability of the electric network that determined by frequency modulation. In modern power system, the concentration of computer control is widely used in each control area, is the secondary frequency compensation of electric power system, namely automatic generation control (AGC).

The occurrence of unexpected failures in the power grid will break the balance between the generation of active power and load. With the development of the power system, single device failures bring a growing loss of generating power grid. Only relying on manually adjust the power generation output will require a longer time to achieve a new balance. Solve these problems. The way is only one that is the techniques of PFC and AGC. It's a good idea to use different logical controls according to its own regulation performance, to maintain the balance of power system supply and demand automatically, thus ensuring the quality of the power system frequency. 


\section{Assessment criteria of PFC}

As an important technical means of frequency control of the power system, PFC is of great significance to the stability of the frequency. Frequency regulation of power system is inherent in the use of system load frequency characteristics, as well as the role of the generator's governor to prevent the system frequency deviation from the standard adjustment method. The grid frequency is determined by the value of generated energy and electricity consumption. If the value of generated energy is equal to the electricity consumption, the grid frequency is stably. If the value of generated energy is greater than the electricity consumption, the grid frequency will increase. If the value of generated energy is less than the electricity consumption, the grid frequency will reduce.

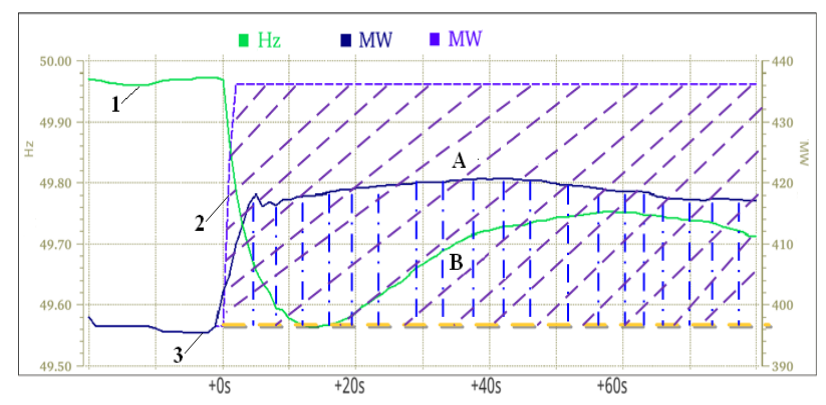

Figure 1. Schematic of PFC's Assessment Criteria

As shown in Figure 1, it's the load response of one unit when grid frequency in the fall. Among them, 1 is grid frequency, 2 is ideal load output, and 3 is actual load output. The shadow area $\mathrm{A}$ is the integral area that the ideal load output minus the initial load output on the assessment time. The shadow area B is the integral area that the actual load output minus the initial load output on the assessment time.

$$
\begin{aligned}
& A=\int_{t_{0}}^{T}\left[P_{s}(t)-P_{0}\right] d t \\
& B=\int_{t_{0}}^{T}\left[P_{r}(t)-P_{0}\right] d t
\end{aligned}
$$

$t_{0}$ is the starting time that the frequency deviation over of the dead band, frequency deviation dead band generally taken to be $0.033 \mathrm{~Hz} . P_{0}$ is the unit load at $t_{0}$ moment. $P_{r}(t)$ is the unit actual load curve. $P_{s}(t)$ is the unit ideal load curve. Calculated $P_{s}(t)$ as following formula

$$
\begin{gathered}
P_{s}(t)=P_{0}+R_{i}\left(t-t_{0}\right), t-t_{0} \leq \frac{P_{\max }-P_{0}}{R_{i}} \\
P_{s}(t)=P_{\max }, t-t_{0}>\frac{P_{\max }-P_{0}}{R_{i}}
\end{gathered}
$$

Among them, $R_{i}$ represents the unit ramp rate. After calculating the shadow area A and B, PFC assessment indicators equal to

$$
\eta=\frac{A}{B} \times 100 \%
$$

Unit PFC assessment rules as follows, if $\eta>0$, it's determined to be correct FM operation. If $\eta \geq \eta_{\min }, \eta_{\min }$ is the threshold value, it is determined to meet the requirements of PFC performance.

According to the statistics of IEEE, the characteristic time constants of PFC in power system is about 10 seconds, it is fast response to the changes of system frequency. Thermal power unit primary frequency regulation effect of time is short. Because of the different heat storage capacity, the action time of PFC to different types of thermal power unit is 0.5 to 2 minutes inequality. PFC is adjusted by the control methods according to differ characteristic, its advantage is the adjustment of all units only with a system frequency related, the interaction of units is small. According to the characteristics of PFC, it's known that the function of PFC control is, to automatic balance the first 
load component, namely those fast, amplitude smaller, random load, and to buffer the abnormal situation of load mutation.

Generally speaking, the PFC in domestic most power plants adopts the power circuit correction (CCS) and speed governing side feedforward (DEH) control strategy. DEH is implementation stage, difference, open loop control, ensure frequency compensation's rapidity, CCS is correction stage, no difference, closed loop control, ensure the accuracy and persistent of frequency compensation. In order to guarantee the quality of regulation, speed and power signal of PFC have been done by selecting midvalve of three same signals, to be shared with DEH and CCS system, to ensure the consistency of judgment on both sides of the signal.

\section{Performance standard of AGC}

AGC is one of the important measures to regulate the frequency and ensure its security and economic operation. PFC is a kind of important way of the frequency control system, but, because of its attenuation characteristic and differ regulation, can't to control the system frequency depending solely on it. In order to maintain the stabilization of frequency, the regulation capability of each control area is evaluated and restricted by control performance standard. To realize zero error output adjustment of frequency, it must rely on the frequency of the AGC. The AGC mainly calculated on area control error (ACE), it equal to

$$
A C E_{i}=K_{i}\left(f-f_{0}\right)+\left(P_{t i e, \mathrm{r}}-P_{t i e, s}\right)=K_{i} \Delta f+\Delta P_{t i e, i}
$$

Among them, $f$ is the actual frequency. $f_{0}$ is the rated frequency. $K_{i}$ is the frequency bias coefficient. is the Tie-line planning power, power flows to the region is positive. When the grid frequency fluctuations occur, the regional power generation $\Delta P_{i}$ that should be adjusted need to satisfy the following formula

$$
\int A C E_{i} d t+\Delta P_{i}=0
$$

Because the adjustment way of AGC for system frequency is no deviation, so, when $A C E_{i}=0$, the PFC is over, $\Delta P_{i}=0$.

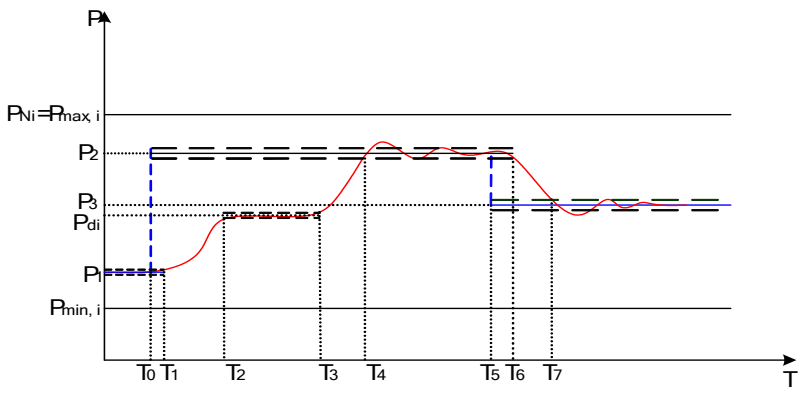

Figure 2. Typical AGC unit set point control process

The above is AGC control of power grid. In terms of one specific unit, adjustment rate, adjustment accuracy and response time is three main criteria of AGC assessment. As shown in Figure 2, it is a set point control process of a typical AGC unit. $P_{\min , i}$ is the lower limit of the unit adjustable output, $P_{\max , i}$ is the upper limit of its adjustable output, $P_{N i}$ is its rated output, $P_{d i}$ is a critical power point of its start and stop grinding. The whole process can be described as, T0 time ago, the unit work stably near the output P1. T0 time, AGC control program sent power command $\mathrm{P} 2$ to unit, the unit start up. To the time T1, unit reliable across the P1 adjustment dead zone, and to the T2 time, unit work into the rev grinding range. Until T3 moment, the rev grinding process is over; the unit continues to raise its power. To T4 time, unit first time work into the dead zone adjustment range, then near P2 unit adjust slightly and has a stable value. Until T5 time, AGC control program sent a new unit set point command, the power is P3, and unit began to a new work. 
3.1. Adjustment rate. According to the requirement of Dispatch center, In general, the rate of Pulverizing System and drum boiler unit is $1.5 \%$ of unit's rated active power, thermal power units with intermediate storage pulverizing system is $2 \%$ of unit's rated active power, circulating fluidized bed coal-fired units is $1 \%$, supercritical once-through boiler unit is $1.0 \%$.

3.2. Adjustment accuracy. It is the difference between the actual output of unit and the set point of EMS when unit work stably after a response, permissible deviation is $1 \%$ of unit's rated active power.

3.3. Response time. Response time refers to, after the EMS system send commands, on the basis of the original output point, the time that the output of generating unit reliably adjust to across adjusting dead zone to need. AGC response time of thermal power unit should be less than 1 minute.

When the unit work in AGC mode, the unit load demand is the sum that the value through rate limitation of AGC command and the frequency modulation. In CCS mode, the turbine control according to the bias that given value and the actual value of load to adjust can realize quick response. Due to the response of boiler combustion have larger delay and inertia, electric load changes quickly when the turbine valve change, so, when the load demand have a change, the turbine valve should adopt a appropriate action valve to make full use of the boiler heat storage in the allowed range of main steam pressure to speed up unit initial load response speed. Technical scheme mainly concentrated in the turbine side.

\section{Problems and solutions}

4.1. Misoperation and Transformation. Contrast PFC assessment data of grid to observe changes in the load history curve DCS found that some conditions, one frequency modulation occur on power plant, but grid side there is no frequency modulation. For example, one $300 \mathrm{MW}$ thermal unit, one day operation number of grid is 15 times, and the DCS of power plant unit records the actual number nearly one thousand times, disoperation is extremely high. Therefore, the root of the problems of PFC signal source is not unified, grid assessment according to the calculated frequency signal which from PMU, CS and DEH control of unit used for speed or electrical measurement frequency, between different source, therefore thermal unit need to signal homologous improvement, ensure $\pm 2 \mathrm{rpm}( \pm 0.033 \mathrm{~Hz})$ action. The misoperation of PFC will affect AGC's adjustment accuracy and adjustment rate.

In order to solve this problem, units need to signal transformation, in order to realize the unification of the controlled object.

4.2. Reverse Adjustment and Logic Optimization. At the same time, PFC and AGC have reverse adjustment problem, cause in extreme cases, the performance of the two are not guaranteed. As shown in Figure 3, AGC demand is in growth, but PFC is reducing, there are two different direction adjustments. Among them, lis AGC demand, 2 is load demand after the rate limit, 3 is actual load, 4 is PFC power compensation.

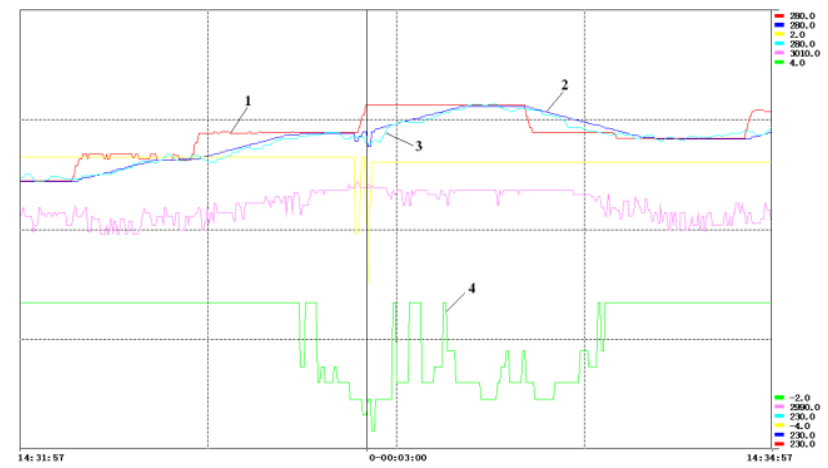

Figure 3. Schematic of PFC's Assessment Criteria 
Because PFC is a priority in AGC, therefore, the unit must firstly ensure that the action effect of PFC, so, the control logic optimization is shown in Figure 4. When turbine speed (grid frequency) deviation is over $\pm 2 \mathrm{rpm}( \pm 0.033 \mathrm{~Hz})$, the turbine demand must be blocked increase or decrease.

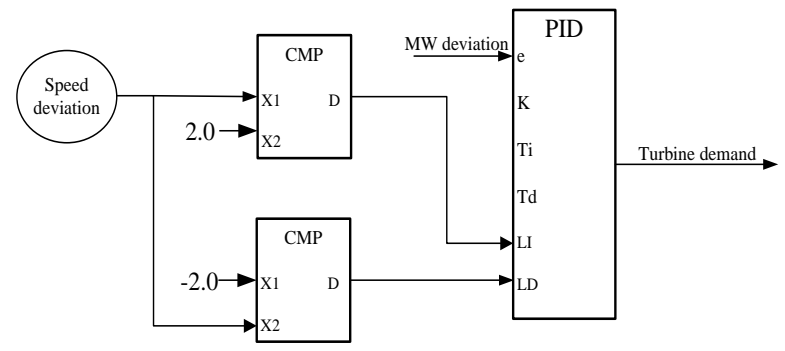

Figure 4. Schematic of PFC's optimal control logic

\section{Actual operating results}

For example, one 300MW subcritical coal-fired power plant, its rated speed is 3000rpm, speed governor droop is $5 \%$, power compensation quantity is $24 \mathrm{MW}$, and AGC adjustment rate is $7 \mathrm{MW} / \mathrm{min}$. After signal technological transformation and control logic optimization, the performance index of PFC and AGC have been effectively improved.

5.1. Effectively reduce PFC's Malfunction number. From the Figure 5, it can clearly be seen that a significant reduction in the number of PFC action, means that interference with the AGC has been effectively controlled. Among them, 1 is action number before the optimization of PFC, 2 is action number after the optimization of PFC.

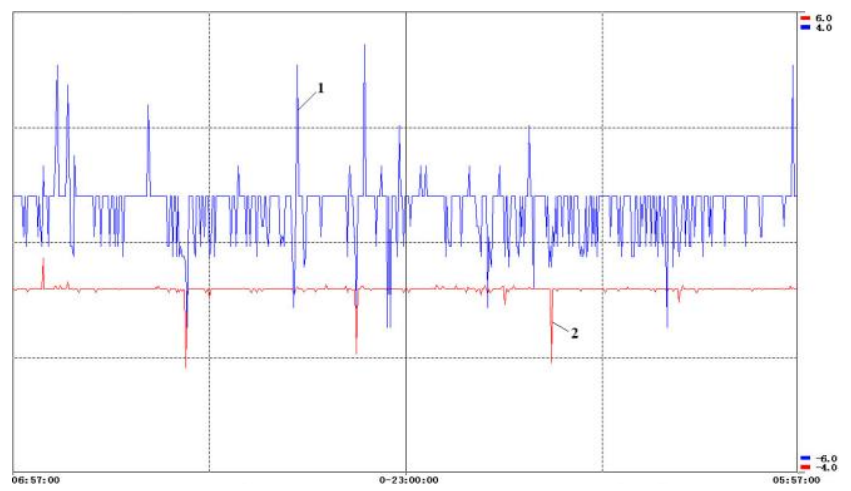

Figure 5. Curve before and after the optimization of PFC

PFC transformations significantly reduce the number of operations, not only reduces the impact on the AGC control performance, while the impact of the damper unit has also been effectively controlled.

5.2. Significantly Improve AGC Index. From the data in Table 1 and Table 2, it can clearly be seen, because the PFC invalid actions were under control, the indicators of AGC are improved.

The value of regulation speed $\mathrm{K} 1$ change from less than 1 to $1.2,1.2$ is the highest value to this index. The comprehensive performance indexes are slightly higher. In particular, the adjustment accuracy index $\mathrm{K} 2$ is raised by $20 \%$, from failure becomes excellent.

Table 1. AGC's Assessment Indicators before Transformation

\begin{tabular}{|c|c|c|c|c|}
\hline Date & K1 & K2 & K3 & Kp \\
\hline $03-13$ & 1.119 & 0.964 & 1.701 & 1.834 \\
\hline $03-12$ & 1.081 & 0.958 & 1.717 & 1.778 \\
\hline $03-11$ & 1.094 & 0.972 & 1.685 & 1.792 \\
\hline $03-10$ & 1.122 & 0.993 & 1.709 & 1.902 \\
\hline $03-09$ & 1.057 & 0.938 & 1.690 & 1.675 \\
\hline $03-08$ & 1.131 & 1.001 & 1.698 & 1.921 \\
\hline
\end{tabular}


Table 2. AGC's Assessment Indicators after Transformation

\begin{tabular}{|c|c|c|c|c|}
\hline Date & K1 & K2 & K3 & Kp \\
\hline $06-22$ & 1.2 & 1.201 & 1.747 & 2.517 \\
\hline $06-21$ & 1.2 & 1.145 & 1.732 & 2.380 \\
\hline $06-20$ & 1.2 & 1.092 & 1.729 & 2.265 \\
\hline $06-19$ & 1.2 & 1.137 & 1.747 & 2.384 \\
\hline $06-18$ & 1.2 & 1.104 & 1.739 & 2.305 \\
\hline $06-17$ & 1.2 & 1.127 & 1.743 & 2.356 \\
\hline
\end{tabular}

\section{Conclusions}

As two important technical means of frequency control of the power system, PFC and AGC are of great significance to the stability of the frequency. In order to better quality of frequency modulation, need to AGC combined with PFC. When big power grid frequency fluctuation occur, PFC should act immediately, and AGC should be according to the size of the ACE, dynamic adjustment the number of units into emergency mode, as soon as possible to return to $50 \mathrm{~Hz}$. That is, when there is a large power grid frequency deviation, ACE load range at the emergency regulation area, to be based on the magnitude of the load is missing the appropriate amount of operating units, in order to quickly make up the power shortfall. Meanwhile, in order to maintain the stabilization of frequency, the regulation capability of each control area is evaluated and restricted by control performance standard. The update and field tests of generator PFC and AGC function should be carried out.

\section{References}

[1] D.R.Coyghanowr, Process Systems Analysis and Control, McGraw-Hill, New York, 1991

[2] YIN Feng. Test and research on CCS-joined primary frequency regulation of thermal power units [J]. Electric Power, 38(3), 2005

[3] WANG Zhen-yi, XIE Yi-gong, YIN Cheng-quan, et al. Research on the coordination of AGC and primary frequency regulation based on CPS [J]. Power System Protection and Control, 37(19), 2007

[4] CHEN Liang, CHEN Hui-kun. Analysis on primary frequency regulation of generator units in Guangdong Power System [J]. Guangdong Electric Power, 21(8), 2007

[5] ZHU Wei, TAN Xi-yi, TANG Ying-jie, et al. Analysis and research on primary frequency modulation of the turbine generation unit[J]. Automation of Electric Power Systems, 32(24), 2008

[6] M. Zhuang, D.P. Atherton, Automatic tuning of optimum PID controllers, IEE ProcD, 140, 1993 Antonio Visioli, A new design for a PID plus feedforward controller, Journal of Process Control, 14, 2004

[7] T. Liu, W.D. Zhang, F. Gao, Analytical decoupling control strategy using a unity feedback control structure for MIMO processes with time delays, J. Process Control, 17 ,2007

[8] K.-H. Park, Z. Bien, D.-H. Hwang, Design of an iterative learning controller for a class of linear dynamic systems with time delay, IEE Proc. - Control Theory Appl. 145 ,1998

[9] Tan, W., Marquez, H.J. \& Chen, T. "Performance assessment of PID controllers", Control and Intelligent Systems, 32, 2004

[10]D.E. Seborg, T.F. Edgar, D.A. Mellichamp, Process Dynamic and Control, seconded., John Wiley \& Sons, New Jersey, 2004. 